REVIEW ARTICLE

\title{
Epigenetic changes in health and disease: DNA Methylation in human development, infection and non-communicable diseases
}

\author{
P. A. J. Perera* \\ Department of Biochemistry, Faculty of Medicine and Allied Sciences, Rajarata University of Sri Lanka, Saliyapura \\ 50008, Sri Lanka
}

Received in revised form:27/02/2017; Accepted:24/01/2018

\begin{abstract}
Following completion of the sequencing of the human genome in 2001 attempts were made to decipher functional units imprinted in the genome, their regulation, and deregulation that caused disease. The genetic and epigenetic information laid down in the genome were recognized and their functions investigated. The coding genes laid down the blueprint for the synthesis of all proteins necessary for functioning while epigenetic markers in the form of methylated $\mathrm{CpG}$ dinucleotides on the genome commanded their expression at appropriate times enabling human to live through its full life cycle. Zygote genome formed following fertilization was found to be globally hypomethylated and was pluripotent. However when it was subjected to cell specific differentiation the genome underwent hypermethylation giving rise to different morphological and functional characteristics. Promoter regions of some of the genes were activated while some others were silenced. These were the dictums of internal and external signals elicited through epigenetic marks laid down in the human genome. Infections and chronic non-communicable diseases such as cancers, cardiovascular diseases etc were found to be the outcome of aberration in genomic DNA methylation. These epigenome changes being reversible act as feasible targets to achieve normalcy. If detected early preventive and curable action could be implemented.
\end{abstract}

Keywords: Genetic and epigenetic information, DNA methylation, development, infection, non-communicable diseases.

\section{HUMAN GENOME}

The human genome comprising of 23 pairs of chromosomes and mitochondrial DNA provide the information necessary for the sustenance of human life throughout the life cycle. The manner in which life conceived as a result of fertilization of an ovum by a sperm progresses to adult life is laid down in the human genome in two different instructional forms, namely the genetic and the epigenetic forms (Brown, 2002).

\section{GENETIC INFORMATION}

The genetic information inherited from the parents provides the blueprint for the synthesis of all proteins necessary for human life while epigenetic information instructs how, where and when genetic information should be used (Robertson, 2001). The intimate relationship between these two forms enable genes to be switched on and off at the appropriate times enabling human to live a full life.

\section{EPIGENETIC INFORMATION}

Cells in the human body are genetically homogenous but structurally and functionally heterogeneous, resulting from differential expression of genes. Many of these differences in gene expression arise during development and are retained during mitosis. Stable alterations of these types are referred to as epigenetic changes (Jaenisch and Bird, 2003).

Epigenetic information appears in DNA following addition and removal of chemical tags attached to DNA and DNA associated protein. It results in stable heritable phenotype changes without alteration of DNA sequence (Berger et al., 2009). Small RNAs too have been implicated in the expression of genes at the level of m-RNA (Feng et $a l ., 2010)$. This is done by short non-coding micro-RNAs binding to untranslated 3'- region of m-RNA causing translational silencing (Cannel et al., 2008).

Epigenetic changes in DNA and protein result in rebuilding of chromatin structure in an open or closed configuration thereby expressing or repressing genes controlling basic cellular processes such as differentiation, proliferation and apoptosis (Gnyszka et al., 2013).

During cell specialization DNA and histones get rearranged. These changes determine how and when different sets of genes are turned on or off. The epigenome controls many of these changes in the genome. Such changes are prominently seen in prenatal life and early postnatal life at the time of cell differentiation and specific tissue formation. These events are influenced by nutrition and environmental factors (Barouki et al., 2012).

Certain dietary components have been recognized to alter genomic and gene specific DNA methylation levels in systemic and target tissues, affecting genomic stability and transcription of tumor suppressor genes and oncogenes. Supportive evidence exists for folate, a key nutritional factor involved in one carbon transfers and this includes methylation reactions. Other bioactive food compounds include alcohol, polyphenols and flavonoids of green tea, phytoestrogen and lycopene (King-Batoon et al., 2008; Lim and Song, 2012). 
Earlier human disease was thought to originate from changes in the base sequence of DNA, infections or toxic environmental agents. However, as of to-day, epigenetic changes and not genetic base sequence changes are considered to be the likely common cause. Currently, increase in the incidence of non-communicable diseases which are rampant in the world is associated with aberrant epigenetic changes in DNA and DNA associated protein. Life style and environmental factors that include smoking (Lee et al., 2013), diet and infectious diseases (Lim and Song, 2012; de Moneri and Lim, 2014) prompt chemical responses. These responses, in turn, often lead to changes in the epigenome, some of which can be detrimental to life, cancer, obesity etc. The ability of the epigenome to adjust to the pressures of life appears to be required for normal human health (Lim and Song, 2012).

Epigenetic marks are reversible but some may be carried from one generation to the other without change (Trerotola et al., 2015). Some epigenetic changes are detrimental to health and these could be reverted back to normal status by taking appropriate measures such as diet, life style and environmental changes that promote reversal of such changes (Wright, 2017).

\section{DNA METHYLATION}

DNA methylation is a stable epigenetic marker (Reik, 2007). It is used by cells to control gene expression. DNA methylation refers to the addition of a methyl $\left(\mathrm{CH}_{3}\right)$ group to the DNA strand itself, often to the fifth carbon atom of a cytosine ring. When gene promoter region on DNA is methylated it typically acts to repress transcription.

In somatic cells, methylation is commonly found in cytosine residue of the $\mathrm{CpG}$ dinucleotide. However in embryonic stem cells methylation is prevalent in non $\mathrm{CpG}$ dinucleotides. In genomic DNA most of the $\mathrm{CpG}$ sites are heavily methylated while $\mathrm{CpG}$ clusters in germ line tissues and promoter regions of somatic cells remain unmethylated (Reik, 2007). In the case of plants and bacteria in addition to cytosine, adenine is also found to be methylated (Vanyushin, 2006, Casadesus and Low, 2006).

DNA methylation regulates many cellular processes, including cellular differentiation and development (Erhlich, 2003), transcription, chromatin structure, X-chromosome inactivation, genomic imprinting and chromosome stability (Meng et al., 2015).

\section{PROTO-ONCOGENES AND SUPPRESSOR GENES}

Proto-oncogenes produce proteins which are usually growth promoters that normally enhance cell division or inhibit normal cell death. The mutated forms of these genes referred to as oncogenes result in uncontrollable expression resulting in cancer. The second group, called tumor suppressor genes make proteins that normally prevent cell division or cause cell death (Lodish et al., 2000). It is the rational interplay between the proto-oncogenes and the suppressor genes that enable the human to maintain healthy life. Activation or inactivation of these genes could also result from epigenetic changes, but these would be reversible unlike genetic base sequence changes.

\section{DNA METHYLASE}

DNA methylase transfers a methyl group from S-adenosylL-methionine to the 5-position of cytosine residues of $\mathrm{CpG}$ dinucleotide in DNA and plays an important role in maintaining genome stability. Four DNA methylases referred to as DNA methyltransferases DNMT1, DNMT3A, DNMT3B and DNMT3L have been identified. Of these the first three are essential for mammalian development (Cheng and Blumenthal, 2008; Dhe-Paganon et al., 2011; Jin and Robertson, 2013). DNMT3A and DNMT3B enzymes have high affinity for non-methylated DNA and are necessary for methylation of genome following fertilization whereas DNMT1 maintains this pattern during chromosome replication.

Aberrant expression of DNA methylases and disruption of DNA methylation patterns are associated with many forms of cancer but the exact mechanisms remains elusive. This is despite DNA repair mechanisms that have evolved to recognize and repair both exogenous and endogenous DNA insults. Promoters of tumor suppressor genes and DNA repair genes are frequently hypermethylated in a variety of tumors (Jin and Robertson, 2013).

DNMT1 codes for the maintenance methyltransferase and, being the most abundant form in mammalian cells, is highly expressed during the $\mathrm{S}$ phase of the cell cycle. It faithfully maintains the DNA methylation marks in somatic cells after each replication cycle (Detich, et al., 2001). DNMT1's preference for hemi-methylated CpG sites compared to unmethylated sites is thought to form the high fidelity maintenance of the epigenetic code during replication (Adams \& Lindsay, 1993; Bashtry et al., 2012). DNMT1 is highly expressed in cancer cells and is associated with cellular transformation (Subramaniam et al., 2014). It is a potential anti-cancer target. Complete inactivation of DNMT1 in human colorectal carcinoma cell lines causes genomic instability, defective chromatin as well as mitotic arrest (Chen et al., 2007).

Using this information, an attempt was made to use derivatives of 5-aza-2'-deoxycytidine as a competitive inhibitor of DNMT1, in the treatment of leukemia patients. It is perceived that lack of knowledge about protein structure, molecular mechanism of regulation, plethora of interacting and regulatory protein factors and high risk of potential mutagenesis and carcinogenicity may have resulted in its poor application (Dhe-Paganon et al., 2011).

\section{MOLECULAR SWITCH}

Methylation/demethylation of cytosine residue on $\mathrm{CpG}$ dinucleotide in the promoter region of DNA acts like a molecular switch. It is on when cytosine is non-methylated and off when cytosine is methylated. In the switched-on position transcription is active. The change is normally 
permanent and unidirectional and does not allow reversion to stem cells or to other cell types (Wu and Sun, 2006).

DNA methylation stably alters the expression of genes when cells differentiate from embryonic stem cells to tissue specific cells. Progression from embryonic stem cells into differentiated progeny requires long lasting changes in gene expression (Marchal and Miotto, 2015). These changes are accompanied by remarkable changes in morphology and function. During differentiation, genes associated with stem cell self renewal are silenced and cell type specific genes undergo transcriptional activation. DNA methyltransferases DNMT3A and DNMT3B play an important role.

\section{DEMETHYLATION}

Demethylation is carried out by demethylases that remove methyl groups from DNA and proteins, particularly histones. Demethylase enzymes are important in epigenetic modification mechanisms. The demethylase proteins alter the genome by controlling the methylation levels that occur on DNA and histones and, in turn, regulate the chromatin state at specific gene loci within organisms.

Demethylation of DNA is carried out either actively or passively. The active process occurs through hydroxylation of the methyl groups followed by their removal (Wu and Zhang, 2017). A base excision repair mechanism has also been proposed to explain active global demethylation. Other mechanisms too have been postulated but require further studies (Chen and Riggs, 2011).

Passive DNA demethylation could occur through multiple mechanisms during DNA replication where failure of DNA methyltransferase (DNMT) action results in loss of 5-methyl cytosine. These include down-regulation of DNMT enzymes, cytosolic localization of DNMT, impairment of DNMT recruitment on DNA, decrease of the DNMT substrate S-adenosyl L-methionine and inhibition of DNMT enzymatic activity (Wu and Zhang, 2014).

\section{DEVELOPMENT}

Immediately after fertilization zygote genome undergoes global depletion of DNA methylation reaching the lowest level at the time of implantation. This enables embryonic stem cells to become pluripotent. At this stage DNMT1 is localized in the cytoplasm and not in the nucleus (Cardoso and Leonhard, 1999). Following implantation global remethylation occurs enabling DNA methylation patterns to be established with the help of DNA methylases DNMT3A DNMT3B. At this stage cell specific DNA methylation patterns develop establishing differentiated cell forms (Lee et al., 2013). Following cell differentiation, cell division takes place where methylation sites are maintained with the help of DNA methylase DNMT1.

Cells in different tissues perform specialized functions. They all have essentially the same genome in its nucleus but they differ in the way different sets of genes are switched on or off. Specialized cells in the eye turn on genes that make proteins enabling to-detect light while specialized cells in the blood make proteins that carry oxygen to different parts of the body. The basis of this difference lays in the epigenetic code, bulk of which is encoded as a methyl mark on a specific DNA sequence CpG dinucleotide (DhePaganon et al., 2011). Nearly half of all CpG dinucleotides in the genome are methylated. The variation in methylation pattern determines the cell type (Razin et al., 1984).

During embryonic development epigenetic patterns change dramatically due to biological programming and are essential for physiological changes that follow it. These changes are the result of alteration in gene expression arising from switching on/off of molecular switches.

Developmental changes in the expression of globin chains of hemoglobin is observed as the human life cycle proceeds from embryonic to fetal and then to adult life(Mavilio et al., 1983). This phenomenon is the result of under-methylation of globin genes at different times accompanied by their expression in the respective erythroblasts. It is reported that at 6 weeks erythroblasts produce essentially embryonic $\varepsilon$ globin chains, at 12 weeks erythroblasts synthesize largely fetal $\gamma$ globin chains and the adult erythroblasts synthesize almost exclusively $\beta$ globin chains. In all phases of ontogenic development a strong correlation exists between DNA hypomethylation in the close flanking sequences of globin chains and their expression (Mavilio et al., 1983).

\section{GROWTH}

It refers to somatic cell division where 'mother cell' divides equally to two 'daughter cells' with composition identical to the mother cell. Each somatic cell goes through phases G1, S and G2 of cell cycle before undergoing cell division in the mitotic phase. Any environmental damage to DNA at the entry point in G1 phase will be subjected to scrutiny by P53 suppressor gene protein and cells laden with unrepaired DNA will undergo 'apoptosis', natural cell death (Efeyan and Serrano, 2007). During the S phase, DNMT1 is active and introduces methylation tags at the appropriate $\mathrm{CpG}$ dinucleotides on the new replicated DNA identical to the parental DNA.

The duration of cell cycle in each tissue will be determined by the body requirement imprinted in the human genome, the nutritional status and the factors that influence its metabolism via cell signaling. According to Holiday and Pugh (1975) the number of cell cycles undergone or the status of the biological clock is reflected in the number of methyl groups inserted into the $\mathrm{CpG}$ dinucleotides in the repetitive DNA sequences during cell division.

\section{AGING}

Aging invloves a slow time-dependent decline of a set of biological functions (Lopez-Otin et al., 2013). One such functional decline is the decline of resting metabolism. Another is reduced stability of chromosomal epigenetic patterns. Alterations of the epigenome in adult somatic tissues may reflect aging associated deleterious states. These include accumulation of genomic damage that 
lead to chromosomal instability and telomere shortening. Reactive oxygen species induce damage to mitochondrial function and reduce energy production, stem cell depletion, accumulation of damaged protein leading to senescence and changes in intercellular communications, age related effects of insulin and IGF-1 signaling pathways and alteration of the epigenome (Lopez-Otin et al., 2013). Aging and age-related diseases are associated with changes in 5-methylcytosine content in DNA and are generally characterized by genome-wide hypomethylation and promoter specific hypermethylation. These changes contribute towards age related pathologies, such as cancer, neurodegenerative diseases, atherosclerosis, inflammation, osteoarthritis etc.

Another age-related disease is obesity which is a risk factor predisposed to development of non-communicable diseases (Perera and Edirisinghe, 2016).. With aging, from middle age to old age, there is accumulation of truncal fat which is shared between subcutaneous adipose tissue and visceral adipose tissue. In old age fat is redistributed outside fat depots accumulating in bone marrow, muscle, liver and other ectopic sites. Presence of excess dysfunctional depot fat appears to accelerate onset of multiple age related diseases such as diabetes, hypertension, cancer, atherosclerosis etc. (Perera and Edirisinghe, 2016). Gene expression in abdominal subcutaneous tissue differs from intra-abdominal visceral adipose tissue (Vohl et al., 2004). There is evidence to suggest that although primary characteristics of abdominal tissues in subcutaneous and visceral compartments are similar some genes expressed are functionally different. This difference is explained in the co-morbidities observed with visceral fat in abdominal obesity. It is reported that DNA methylation is involved in regulation of depot specific gene expression (Gehrke et al., 2013). According to Ross et al. (2013), impairment of brain development and its aggravation in aging are transferable from mother to the child through germ line mitochondrial DNA mutations. Unlike hereditary genetic changes epigenetic changes are reversible and therefore appear as primary targets for therapeutic intervention.

\section{LIFE SPAN}

In many organisms calorie restriction can extend the life span and also contribute towards delaying the onset of ageassociated changes (Sohal and Forster, 2014). In animals, calorie restriction is reported to reduce metabolic rate and oxidative stress, improve insulin sensitivity and alter neuroendocrine and sympathetic nervous system functions (Heilbron and Ravussin, 2003).

It has been shown that calorie restriction produce coordinated and adaptive stress responses at cellular and whole organism level. These responses are elicited via modulation of epigenetic mechanisms, signaling pathways and cell to cell interactions. The epigenetic mechanisms include DNA methylation and post-translational histone modification. The overall effect of adaptive responses is an increased resistance to subsequent stress leading to delaying age-related changes and longevity. Exercise with or without calorie restriction and use of plant polyphenols are the avenues available to further improve the situation (Ribaric, 2012). Physical activity is associated with higher methylation in a class of repeated sequences highly repeated in the human genome. Further, low methylation of these repetitive elements is associated with inflammatory response as well as chromosomal instability (Zhang et al., 2011).

In the fruit fly Drosophila, a gene has been discovered that helps to determine its life span. A gene named Indy in it when mutated extends its life span. The protein encoded by this gene transports and recycles metabolic byproducts. Defect in the gene produces a protein that renders transport of metabolites linked to the TCA cycle less efficient and thereby limit combustion and energy production. This mimics a situation equivalent to energy restriction. In the fruit fly the ody functions as if the fruit fly was dieting, even though it's eating habits is unchanged. Mutations in Indy, a plasma membrane transporter of Krebs cycle intermediates appear to create a metabolic state that mimics caloric restriction, which extends life-span (Rogina et al., 2000). DNA methylation and DNMTs are considered as likely regulators of health and life span. Telomeres, free radicals, DNA repair machinery, mitochondrial enzymes, energy etc. are considered as possible targets to extend human health life span.

\section{INFECTION}

Humans respond to infection by initiating inflammatory and immune responses whereby infecting organism is cleared from the system (Nicholson, 2016) In cases of resistance to such actions, pathogens are seen to develop mechanisms to modify host cell functionality to promote their survival and, in the case of intracellular pathogens, to generate a suitable environment for them to replicate within the host. This includes modification of transcriptional profile and protein content of the host they infect. Recent studies highlight host chromatin and other epigenetic regulators as targets of pathogens. Many of these changes are short-lived but several studies indicate that pathogens can induce long term heritable changes that are essential for pathogenesis of infectious diseases and persistence of pathogens within the host (de Monerri and Kim, 2014). In 1985, Jähner and Jaenisch showed that integration of viral DNA into host DNA induced local changes in DNA methylation, resulting in transcriptional silencing which contributes to viral latency by the maintenance of proviral DNA in silenced regions.

Leishmaniasis is an infection spread to humans by parasite Leishmania transmitted via sandflies of the Phlebotomus, Lutzomyia and Psychodopygus species through a blood meal (Siriwardana et al., 2012). Leishmania donovani infection causes epigenetic DNA methylation changes in host macrophages compromising its immunity. The epigenetic changes produced in the human DNA result in down regulation of innate immunity, enabling pathogen survival and replication (Marr et al., 2014). 


\section{NON-COMMUNICABLE DISEASES}

Epigenetic changes that occur in response to external stimuli, after remaining stable for sometime can result in long term changes in gene expression leading to pathological dysfunctions (Sharp and Relton, 2017). These dysfunctions give rise to non-communicable diseases that include cardiovascular disease, cancer, chronic respiratory disease and diabetes, the leading causes of death in the world (WHO, 2014). They develop due to interplay of genetic, lifestyle and environmental factors. Many are considered preventable because the most important risk factors, such as tobacco use, alcohol use and obesity, are modifiable.

Human studies have shown associations between epigenomic DNA methylation and several noncommunicable diseases, including cancers, asthma, obesity and Type 2 diabetes (Sharp and Relton, 2017).

\section{CARDIOVASCULAR DISEASE}

Heijmans and co-workers (2008) who studied individuals prenatally exposed to famine during the Dutch Hunger Winter in 1944/45 reported that, compared to siblings of the same sex who were not exposed, these individuals displayed an altered genetic methylation pattern. DNA methylation of the imprinted insulin-like growth factor II gene was shown to be lower. The prevalence of obesity and coronary heart disease in these individuals was higher than that of adults born before or conceived after that period. Low birth weight was also shown to be an independent risk factor for coronary heart disease. These findings are supported by experimental studies, in which the impact of maternal diet during pregnancy on the pattern of DNA methylation of genes pertaining to blood pressure control is seen (Bogdarina et al., 2007). In addition, epigenetic changes on DNA were observed when individuals were exposed to dietary changes, smoking and pollution and these were similar to the epigenetic alterations found in patients with cardiovascular disease. This explains the close link between the postulated cardiovascular risk factors and cardiovascular disease.

Lorenzen et al., (2012) reviewing the different epigenetic modifications likely to cause cardiovascular disease concluded that DNA methylation, histone modification and noncoding RNA represents the molecular substrates which could be acted upon by detrimental environmental stimuli leading to progression of cardiovascular disease. Most of the work accomplished has shed some light on DNA methylation while others require further investigation.

\section{CANCER}

Both genetic and epigenetic changes contribute towards development of cancer. Of these genetic changes are due to gene mutations arising from alteration of base sequences in genomic DNA. Although DNA repair systems have evolved to maintain genomic integrity by countering threats posed by DNA lesions, deficiencies in the repair system cause the lesions to be unrepaired or repaired incorrectly leading to genome instability and mutation. Genome instability refers to a series of chromosomal changes taking place at an accelerated rate in cells derived from the same ancestral precursor. These include simple base changes and structural and numerical chromosome abnormalities (Byani et al., 2007).

On the other hand epigenetic changes that cause cancer are the result of aberrant summative action of methylase and demethylase on genomic DNA (Sadikovic et al., 2008). Therefore, theoretically cancer could be considered to originate from acquisition of abnormal genetic or epigenetic alterations, either singly or in combination of both.

DNA methylation is considered as one of the major epigenetic mechanisms involved in regulation of gene expression and genomic stability is a requirement for its sustenance and together they are necessary for the maintenance of many cellular functions. When DNA methylation is impaired it could cause widespread deregulation of gene expression profiles and disrupt signaling networks that control cell proliferation and its functions and lead to cancer.

\section{ASTHMA}

Asthma is a chronic disease characterized by recurrent breathlessness. It is influenced by heritable modifications and tags that affect the genome without changing the intrinsic DNA sequence. These include DNA methylation, modifications to histone proteins and expression of noncoding RNA. Patients with asthma have alterations in all of these processes. In some cases these differences are linked to disease severity and susceptibility (Brook et al., 2015). In an infant immune study, DNA methylation in cord blood mononuclear cells of children born to asthmatic and non-asthmatic mothers were closely monitored for a decade and compared among themselves and another control group of non-asthmatic group of children. Cord blood mononuclear cell-derived IL- $1 \beta$ levels were also measured. It was reported that methylation in SMAD3, the most connected node within the network of asthma-associated, differentially methylated regions, was selectively increased in children of asthmatic mothers and was associated with childhood asthma risk. Moreover, SMAD3 methylation in neonates with maternal asthma was strongly and positively associated with neonatal production of IL- $1 \beta$, an innate inflammatory mediator (De Vries et al., 2017). The findings demonstrated that childhood asthma begins at birth and involves epigenetic modifications in immunoregulatory and pro-inflammatory pathways and that maternal asthma influences epigenetic mechanisms that contribute towards its establishment in their children.

\section{TYPE 2 DIABETES}

It has its beginning starting from conception. A fertilized ovum is nurtured by the mother that assimilates nutrients from the food she consumes and processes. If there is disarray in the processing of nutrients or its transport to the growing embryo it may seriously affect its growth and development. In gestational diabetes mother is unable to maintain her blood glucose level within the normal range 
due to lowered insulin receptor sensitivity resulting from elevated plasma free fatty acid level and also partly due to the action of reproductive hormones released (Perera and Edirisighe, 2016). Due to increased activity of placental glucose transporter the embryo is supplied with excess glucose resulting in increased growth and delivery of large for date babies with fetal macrosomia.

\section{ABERRANT DNA METHYLATION}

DNA hypermethylation in the promoter $\mathrm{CpG}$ islands of tumor suppressor genes cause heritable transcriptional silencing, preventing essential functions such as repair of DNA errors, slowing down cell division and inducing malfunctioning cells to apoptosis (Jin and Robertson, 2013).

Hypermethylation of promoter region of DNA repair genes is closely linked to a variety of human tumors including colorectal, breast, lung cancers and glioma. When DNA repair genes such as DNA mismatch repair gene hMLH1, the alkyl repair gene O-6-methylguanineDNA methyltransferase, familial breast cancer gene BRAC1 and Werner syndrome gene WRN are silenced, they lead to cancers laden with growth advantages (Jacinto and Esteller, 2007). These result in cells with unrestricted growth characteristics devoid of growth regulation. Major disruptions are seen in the methylation profiles of malignant cells. There is hypermethylation of gene promoters, global hypomethylation of the genome and increased rate of mutation at methylated $\mathrm{CpG}$ dinucleotides.

In the human genome nearly half of all gene promoters are associated with $\mathrm{CpG}$ enriched regions (0.5-5 kb) called $\mathrm{CpG}$ islands. In normal cells majority of the $\mathrm{CpG}$ islands are not methylated, allowing for transcription of their associated genes. In cancer cells, hypermethylation of promoter regions result in loss of gene expression. In all forms of cancer, aberrant promoter hypermethylation exists. Cells susceptible to develop cancer have defective cell cycle regulators, DNA repair genes, genes associated with apoptosis, hormonal regulation, detoxification, metastasis, angiogenesis and others (Sadikovic et al., 2008).

Ganepola et al. (2014) have tested blood seeking the presence of hypermethylated promoters of tumor suppressor genes in cancer patients with the intention of using them as early biomarker detectors of cancer. The results appear to be promising.

\section{ABERRANT DNA HYPOMETHYLATION}

DNA genomic hypomethylation is the second type of DNA methylation defect that is reported to cause cancer. It is commonly found in solid tumors such as prostate cancer, hepatocellular cancer and cervical cancer and also in hematological cancer such as leukemia. Aberrant hypomethylation is hypothesized to activate oncogenes, increasing genomic instability etc. In addition epigenetic global hypomethylation has also been found to cause genome instability leading to cancer (Lim and Maher, 2010; Jiang et al., 2008).
Mutational gene activation, such as deamination of a methylated cytosine in $\mathrm{CpG}$ dinucleotide causing cytosine to thymidine transitional mutation in genes such as P53 and LDL receptor is another alternate mechanism reported in cell transformation.

\section{APPLICATIONS OF EPIGENOMICS}

During the time of differentiation and specific tissue formation organisms are highly sensitive to environmental factors, such as nutrients, chemicals, drugs, infection and other stressors. Such exposures could induce epigenetic changes in the human genome. These changes in the human genome will be different in different tissues although they hold the same genome (Barouki et al., 2012). Prevalence of many of the non-communicable diseases is considered to be the outcome of such interactions. Poor maternal nutrition that affects intrauterine life has been held responsible for the appearance of non-communicable diseases, non-insulin dependent diabetes, hypertension and coronary heart disease in the middle age (Barker, 1990; Feil and Fraga, 2012).

Most of the curative work in clinical practice focused attention on controlling the activities of regulatory proteins that caused aberration of normal cellular function, and most of these were enzymes. This type of intervention requires long term medication and monitoring. Current belief is that epigenetic changes are reversible and that these could be reversed if appropriate action is taken. One possibility is identification and elimination of the etiological factor/s causing it. This includes management of stress, dietary and environmental factors causing such diseases. Such actions require long term management. Its correction at the epigenetic level will be shorter but will require much more research before adoption. However it is a target for future therapy (Kulis and Esteller, 2010). The only feasible application of epigenetics that is currently being tried out is its use as an early marker of cancer and its surveillance (Ganepola et al., 2014).

\section{CONCLUSION}

Review focuses on epigenetic changes that influence human life in health and disease with special reference to DNA methylation. It is invaluable to understand the etiology of such changes with a view to developing strategies to reduce the incidence of non-communicable diseases which affect quality of life and life span. Epigenetic signatures show promise in early diagnostic work (Erfan et al., 2018). The cost of testing genomic-wide methylation is considered similar to current single gene epigenetic tests for imprinting disorders and is less than US \$ 1,000. But a large database with clinical information links is necessary before one could rationally interpret such analysis (Karow, 2018).

\section{REFERENCES}

Adams, R.L.P. and Lindsay, H. (1993). What is hemimethylated DNA? Federation of European Biochemical Societies. 320(3): 243-245. 
Barker, D.J.P. (1990). The fetal and infant origins of adult disease. British Medical Journal. 301(6761): 1111.

Barouki, R., Gluckman, P.D., Grandjean, P., Hanson, M. and Heindel, J. (2012). Development and origin of noncommunicable diseases: implications for research and public health. Environmental Health. 11(1): 42 -51.

Bashtry, P., Jankevicius, G., Samarandache, A., Jurkowska, R.Z., Ragozin, S. and Jeltz, A. (2012). Specificity of DNMT1 for methylation of hemi-methylated CpG sites resides in its catalytic domain. Chemistry and Biology. 19: $572-578$.

Bayani, J., Selvarajah, S., Maire, G., Vukovic, B., AlRomaiih, K., Zielenska, M. and Squire, J.A. (2007). Genomic mechanisms and measurement of structural and numerical instability in cancer cells. Seminars in Cancer Biology. 17: 5-18.

Berger, S.L., Kouzarides, T., Shiekhattar, R. and Shilatifard, A. (2009). An operational definition of epigenetics. Genes and Development. 23(7): 781-783.

Bogdarina, I., Welham, S., King, P.J., Burns, S.P. and Clark, A.J. (2007). Epigenetic modification of the renin-angiotensin system in the fetal programming of hypertension. Circulation Research. 100(4): 520-526.

Bostick, M., Kim, J.K., Esteve, P.O., Clark, A., Pradhan, S. and Jacobson, S.E. (2007). UHRF1 plays a role in maintaining DNA methylation in mammalian cells. Science. 317: 1760-1764.

Brown, T.A. (2002). Chapter 1. The human genome. In Genomes. $2^{\text {nd }}$ edition. Oxford: Wiley-Liss.

Cannel, I.G., Kong, Y.W. and Bushell, M. (2008). How do microRNAs regulate gene expression? Biochemical Society Transactions. 36(6): 1224-1231.

Cardoso, M.C. and Leonhard, H. (1999). DNA methyltransferase is actively retained in the cytoplasm during early development. Journal of Cell Biology. 147: 25-32.

Casadesus, J. and Low, D. (2006). Epigenetic gene regulation in bacterial world. Microbiology and Molecular Biology Reviews. 70(3): 830-856.

Chen, T., Hevi, S., Gay, F., Tsujimoto, N., He, T., Zhang, B., Ueda, Y. and Li, E. (2007). Complete inactivation of DNMT1 leads to mitotic catastrophe in human cancer cells. Nature Genetics. 39(3): 391-396.

Chen, Z.X. and Riggs, A.D. (2011). DNA methylation and demethylation in mammals. Journal of Biological Chemistry. 286: 18347-18353.

Cheng, X. and Blumenthal, R.M. (2008). Mammalian DNA methyltransferases: a structural perspective. Structure. 16: 341-350.

de Monerri, N.C.S. and Kim, K. (2014). Pathogens highjack the epigenome: a new twist on host-pathogen interactions. The American Journal of Pathology. 184(4): 897-911.

Detich, N., Ramachandani, S. and Szyf, M. (2001). A conserved 3'-untranslated element mediates growth regulation of DNA methyltransferase1 and inhibits its transforming activity. The Journal of Biological Chemistry. 276: 24881-24890.

Dhe-Paganon, S., Sayeda, F. and Park, L. (2011). DNA methyl transferase1: regulatory mechanisms and implications in health and disease. International
Journal of Biochemistry and Molecular Biology. 2(1): 58-66.

Efeyan, A. and Serrano, M. (2007). P53: Guardian of the genome and policeman of the oncogenes. Cell Cycle. 6(9): 1006-10.

Ehrlich, M. (2003). Expression of various genes is controlled by DNA methylation during mammalian development. Journal of Cellular Biochemistry. 88: 899-910.

Erfan, A., David, I.R., Laila, C.S., et al. (2018). Genomic DNA methylation signatures enable concurrent diagnosis and clinical genetic variant classification in neurodevelopmental syndromes. American Journal of Human Genetics. 102(1): 156-174. Published online doi: 10.1016/j.ajhg. 2017.12.008.

Fang, M., Chen, D. and Yang, C.S. (2007). Dietary polyphenols may affect DNA methylation. Journal of Nutrition. 137: 223S-228S.

Feil, R. and Fraga, M.F. (2012). Epigenetics and the environment: emerging patterns and implications. Nature Reviews Genetics. 13: 97-109.

Feng, S., Jacobson, S.E. and Reik, W. (2010). Epigenetic reprogramming in plant and animal development. Science. 330: 622-627.

Ganepola, G.A.P., Nizin, J., Rutledge, J.R. and Chang, D.H. (2014). Use of blood-based biomarkers for early diagnosis and surveillance of colorectal cancer. World Journal of Gastrointestinal Oncology. 6(4): 83-97.

Gehrke, S., Brueckner, B., Schepky, A., Klein, J., Iwen, A., Bosch, C.G., Wenck, H., Winnefeld, M. and Hagemann, S. (2013). Epigenetic regulation of depot-specific gene expression in adipose tissue. PLOS ONE. 8(12): e82516. doi:10.1371/journal.pone.0082516.

Gnyszka, A., Jastrzebski, Z. and Flis, S. (2013). DNA methyltransferase inhibitors and their emerging role in epigenetic therapy in cancer. Anticancer Research. 33: 2989-2996.

Heijmans, B.T., Tobi, E.W., Stein, A.D., Putter, H., Blauw, G.J., Susser, E.S., Slagboom, P.E. and Lumey, L.H. (2008). Persistent epigenetic differences associated with prenatal exposure to famine in humans. Proceedings of the National Academy of Sciences of the United States of America. 105(44): 17046-17049.

Heilbron, L.K. and Ravussin, E. (2003). Calorie restriction and aging: review of the literature and implications for studies in human. American Journal of Clinical Nutrition. 78: 361-369.

Holiday, R. and Pugh, J.E. (1975). DNA modification mechanisms and gene activity during development. Science. 187(4173): 226-232.

Jacinto, F.V. and Esteller, M. (2007). Mutator pathways unleashed by epigenetic silencing in human cancer. Mutagenesis. 22(4): 247-253.

Jaenisch, R. and Bird, A. (2003). Epigenetic regulation of gene expression: how the genome integrates intrinsic and environmental signals. Nature Genetics. 33: 245254.

Jähner, D. and Jaenisch, R. (1985). Retrovirus-induced de novo methylation of flanking host sequences correlates with gene inactivity. Nature. 315: 594-597. 
Jiang, L., Gonda, T.A., Gamble, M.V., Salas, M., Seshan, V., Tu, S., Twaddel, W.S., Hegyi, P., Lazar, G., Steele, I., Varro, A., Wang, T.C. and Tycko, B. (2008). Global hypomethylation of genomic DNA in cancer associated myofibroblasts. Cancer Research. 68(23): 9900-9908.

Jin, B. and Robertson, K.D. (2013). DNA methyltransferases (DNMTs), DNA damage repair, and cancer. Advances in Experimental Medicine and Biology. 754: 3-29. doi: 10.1007/978-1-4419-9967-2_1.

Karow, J. (2018). Epigenetic signatures show promise for diagnosing neurodevelopmental disorders. GenomeWeb. Accessed from https://www.genomeweb. $\mathrm{com} /$.

King-Batoon, A., Leszczynska, J.M. and Klein, C.B. (2008). Modulation of gene methylation by genistein or lycopene in breast cancer cells. Environmental and Molecular Mutagenesis. 49: 36-45.

Kulis, M. and Esteller Manel. (2010). DNA methylation and cancer. In: Advances in Genetics. 70: 27-56.

Lee, K.W.K. and Pausaova, Z. (2013). Cigarette smoking and DNA methylation. Frontiers in Genetics. 4(132): $1-11$.

Lim, D.H.K. and Maher, E.R. (2010). DNA methylation: a form of epigenetic control of gene expression. The Obstetrician and Gynaecologist. 12: 37-42.

Lim, U. and Song, M.A. (2012). Chapter 23. Dietary and lifestyle factors of DNA methylation. In: R. G. Dumitrescu and Mukesh Varma (Eds.) Cancer Epigenetics: Methods and Protocols, Methods in Molecular Biology. $863 \mathrm{Pp}$.

Lodish, H., Berk, A., Zipursky, S.L., Matsudaira, P., Baltimore, D. and Darnell, J. (2000). Section 24.2. Proto-oncogenes and tumor suppressor genes. In: Molecular Cell Biology. $4^{\text {th }}$ edition. W H Freeman \& Company, New York.

Lopez-Otin, C., Blasco, A.B., Partridge, L., Serrano, M. and Kroemer, G. (2013). The hallmarks of aging. Cell. 153(6): 1194-1217.

Marchal, C. and Miotto, B. (2014). Emerging concept in DNA methylation: role of transcription factors in shaping DNA methylation patterns. Journal of Cellular Physiology. 230: 743-751.

Marr, A.K., MacIssac, J.L., Jiang, R., Airo, A.M., Kobor, M.S. and McMaster, W.R. (2014). Leishmania donovani infection causes distinct epigenetic DNA methylation changes in host macrophages. PLOS Pathology. 10(10): e1004419.

Mavilio, F., Giampaolo, A., Care, A., Migliaccio, G., Calandrini, M., Russo, G., Pagliardi, G.L., Mastroberardino, G., Marinucci, M. and Peschle, C. (1983). Molecular mechanisms of human hemoglobin switching: selective undermethylation and expression of globin genes in embryonic, fetal, and adult erythroblasts. Proceedings of the National Academy of Sciences of the United States of America. 80(22): 69076911.

Meng, H., Cao, Y., Qin, J., Song, X., Zhang, Q., Shi, Y. and Cao, L. (2015). DNA methylation, its mediators and genome integrity. International Journal of Biological Sciences. 11(5): 604-617. doi:10.7150/ijbs.11218.
Available from http://www.ijbs.com/v11p0604.htm.

Nicholson, L.B. (2016). The immune system. Essays in Biochemistry. 60(3): 275-301.

Oda, M., Oxley, D., Dean, W. and Reik, W. (2013). Regulation of lineage specific hypomethylation in mouse trophectoderm. PLoS One. 8(6): e68846.

Ordovas, J.M. and Smith, C.E. (2010). Epigenetics and cardiovascular disease. Nature Reviews Cardiology. 7(9): 510-519.

Perera, P.A.J. and Edirisinghe, E.M.I. (2016). Body fat and its influence on health and disease. Ceylon Journal of Science. 45(2): 15-23.

Razin, A., Webb, C., Szyf, M., Yiseraeli, J., Rosenthal, A., Naveh-Many, T., Sciaky-Gallili, N. and Cedar, H. (1984). Variation in DNA methylation during mouse cell differentiation in vivo and in vitro. Proceedings of the National Academy of Sciences of the United States of America. 81: 2275-2279.

Reik, W. (2007). Stability and flexibility of epigenetic gene regulation in mammalian development. Nature. 447: 425-432.

Ribaric, S. (2012). Diet and aging. Oxidative Medicine and Cellular Longevity. 2012: Article ID 741468. 20 pages. doi:10.1155/2012/741468.

Robertson, K.D. (2001). DNA methylation, methyltransferases, and cancer. Oncogene. 20: 31393155.

Rogina, B., Reenan, R.A., Nilsen, S.P., and Helfand, S.L. (2000). Extended life-span conferred by co- transporter gene mutations in Drosophila. Science. 290(5499): 2137-2140.

Ross, J.M., Stewart, J.B., Hagstrom, E., Brene, S., Mourier, A., Coppotelli, G., Freyer, C., Lagouge, M., Hoffer, B.J., Olson, L. and Larsson, N.G. (2013). Germline mtDNA mutations aggravate aging and can impair brain development. Nature. 501(7467): 412-415.

Sadikovic, B., Al-Romiah, K., Squire, J.A. and Zielenska, M. (2008). Cause and consequences of genetic and epigenetic alterations in human cancer. Current Genomics. 9(6): 394-408.

Sharp, G.C. and Relton, C.L. (2017). Epigenetic and noncommunicable diseases. Epigenomics. 9(6): 789-791.

Siriwardana, H.V.Y.D., Chandrawansa, P.H., Sirimanna, G. and Karunaweera, N.D. (2012).

Leishmaniasis in Sri Lanka: a decade old story. Sri Lanka Journal of Infectious Diseases. 2(2): 2-12.

Sohal, R.S. and Forster, M.J. (2014). Calorie restriction and the aging process: A critique. Free Radical Biology and Medicine. 73: 366-382.

Subramaniam, D., Thombre, R., Dhar, A. and Anant, S. (2014). DNA Methyltransferases: a novel target for prevention and therapy. Frontiers in Oncology. 4: 80. Published on line: doi: 10.3389/fonc.2014.00080.

Trerotola, M., Relli, V., Simeone, P. and Alberti, S. (2015). Epigenetic inheritance and the missing heritability. Human Genomics. 9:17. Published on line doi:10.1186/ s40246-015-0041-3

Ulrey, C.L., Liu, L., Andrews, L.G. and Tollefsbol, T.O. (2005). The impact of metabolism on DNA methylation. Human Molecular Genetics. 14 (Special No.1): R139-R147. 
Vanyushin, J. (2006). DNA methylation in plants. Current Topics in Microbiology and Immunology. 301:67-122.

Vohl, M.C., Sladek, R., Robitaille, J., Gurd, S., Marceau, P., Richard, D., Hudson, T.J. and Tchernof, A. (2004). A survey of genes differentially expressed in subcutaneous and visceral adipose tissue in men. Obesity Research. 12(8): 1217-1222.

WHO. (2014). WHO Global status report on noncommunicable diseases. World Health Organization. Geneva, Switzerland. P1-51.

$\mathrm{Wu}$, H. and Sun, Y.E. (2006). Epigenetic regulation of stem cell differentiation. Pediatric Research. 59(4): 21R-25R. doi: 10.1203/01.pdr.0000203565.76028.2a.

Wu, H. and Zhang, Y. (2017). TET- mediated active DNA methylation mechanism, function and beyond. Nature Review Genetics. 18: 517-534.

Wu, H. and Zhang, Y. (2014). Reversing DNA methylation: mechanisms, genomics and biological functions. Cell. 156(1-2): 45-68. 